\title{
Utilisation des microhabitats par le vairon, le goujon et la loche franche dans trois rivières du sud-ouest de la France
}

\author{
S. Mastrorillo' \\ F. Daubal \\ A. Belaud ${ }^{1}$
}

Mots clés : profils de microhabitat, échantillonnage ponctuel d'abondance, Phoxinus phoxinus, Gobio gobio, Barbatula barbatula.

L'utilisation des microhabitats par le vairon, Phoxinus phoxinus (L.), le goujon, Gobio gobio (L.) et la loche franche, Barbatula barbatula (L.) a été étudiée en conditions d'étiage d'hiver et d'été dans trois rivières du sud-ouest de la France : l'Ariège, la Nive et le Gave de Pau. Les poissons ont été échantillonnés par pêche électrique selon la méthode de l'Echantillonnage Ponctuel d'Abondance. Onze variables du milieu ont été prises en compte pour décrire l'environnement ponctuel des poissons. Pour chaque rivière, les associations interspécifiques et espèces-variables du milieu ont été calculées et comparées en fonction de la saison.

Des variations saisonnières de l'utilisation du microhabitat et des associations interspécifiques ont été observées sur les trois rivières. La nature du substrat et la présence d'abris végétaux jouent un rôle important dans la microrépartition du vairon, du goujon et de la loche franche. Ces structures offrent aux poissons des habitats de refuge et de nutrition privilégiés.

\section{Microhabitat use by minnow, gudgeon and stone loach in three rivers in southwestern France}

Keywords: microhabitat profiles, point abundance sampling, Phoxinus phoxinus, Gobio gobio, Barbatula barbatula.

Microhabitat use by minnow, Phoxinus phoxinus (L.), gudgeon, Gobio gobio (L.) and stone loach, Barbatula barbatula (L.) was studied during winter and summer reduced discharge periods in the Rivers Ariège, Nive and Gave de Pau, southwestern France. Fish samples were collected using point abundance electrofishing and data on eleven environmental variables was obtained simultaneously. Interspecific relationships and species-habitat relationships were determined and compared by season in the three rivers.

The observed seasonal variations of interspecific and species-habitat relationships were related to substrate variables and instream cover which play an important role in the spatial distribution of the three fish species studied.

\section{Introduction}

La répartition des espèces résulte des contraintes biologiques (reproduction, alimentation, ...) mais aussi de contraintes écologiques plus larges qui intègrent diverses variables de l'environnement physique (hydrodynamique, nature du substrat, abris rocheux, couverture végétale) et de l'environnement biologique (interaction des espèces ou des individus).

I. Laboratoire Ingénierie Agronomique, Equipe Environnement Aquatique et Aquaculture, INP -ENSA, 145, avenue de Muret, F-31076 Toulouse Cedex, France.
L'utilisation de l'habitat des cours d'eau d'altitude par les salmonidés (Zone supérieure à truite) a fait l'objet ces dernières années de nombreux travaux (Shirvell \& Dungey 1983, Heggenes 1988, Baran 1995) justifiés par la forte valeur économique et halieutique de ces espèces de poissons, de l'intégrité des milieux qu'elles symbolisent et de la fragilité des écosystèmes aquatiques montagnards qui les hébergent. Dans la partie inférieure de la zone à truite, les salmonidés cohabitent avec des espèces dites accompagnatrices, souvent numériquement dominantes, dans un environnement parfois soumis à des évolutions saisonnières complexes où les réponses des populations de salmonidés ne sont pas toujours en accord avec les prévisions formulées par les modèles. 
L'intensification des travaux sur les relations habitat/espèces accompagnatrices de la truite se révèle donc indispensable pour une approche de l'extension du modèle monospécifique (truite) à un modèle plurispécifique et/ou dans une perspective de substitution au modèle truite dans les zones où les populations de salmonidés sont limitées par de multiples facteurs.

En Amérique du Nord, une attention particulière a été portée sur l'habitat des espèces non-salmonidés («coarse» fishes) (Baltz et al. 1982, Grossman \& Freeman 1987).

L'étude porte sur les trois espèces accompagnatrices de la truite (Salmo trutta L.) - le vairon, Phoxinus phoxinus (L.), le goujon, Gobio gobio (L.) et la loche franche, Barbatula barbatula (L.) (cf Wheeler 1992 pour la nouvelle nomenclature) - dans trois rivières du sud-ouest de la France.

En Europe, de nombreux travaux ont été faits sur le comportement (Kennedy 1981, Stott \& Buckley 1979), sur l'influence des conditions extrêmes climatiques et hydrauliques (Mills 1988, Lobón-Cerviá et al. 1991), sur le régime alimentaire, la croissance et la reproduction (Mann 1980, Wootton et al. 1980, Cui \& Wootton 1989, Przybylski \& Bañura 1989, Copp et al. 1994), sur le parasitisme (Bean \& Winfield 1989) du vairon, du goujon et de la loche franche. Mais il existe peu d'informations sur l'habitat de ces trois espèces (Smyly 1955, Welton et al. 1991, Copp 1992) et encore moins sur la dynamique saisonnière de l'utilisation des microhabitats."

Le but de cette étude est de dégager les relations qui existent entre le vairon, le goujon et la loche franche, puis entre chacune de ces espèces et des caractéristiques de l'environnement dans les trois rivières du sud-ouest de la France (Ariège, Nive et Gave de Pau) et de les comparer en périodes d'étiage hivernal et estival.

\section{Zone d'étude}

\subsection{Descriptions des rivières (Fig. 1)}

Le Gave de Pau a un cours de $189 \mathrm{~km}$ de longueur avant sa confluence avec l'Adour. Tout au long de son cours, on relève la présence de nombreux barrages et. seuils édifiés pour satisfaire divers usages de l'eau (hydroélectricité, irrigation, etc) ou pour stabiliser les berges et les ponts. La physicochimie des eaux du Gave de Pau est influencée par les traversées des villes de Lourdes et Pau, du complexe chimique de Lacq-Artix et du développement intensif dans son bassin versant de la monoculture du maïs.
La Nive, dernier affluent rive gauche de l'Adour, a une longueur de $75 \mathrm{~km}$. Son bassin versant couvre environ $1000 \mathrm{~km}^{2}$; il est soumis à un climat de caractère océanique prononcé avec des températures douces et des amplitudes faibles. Ces eaux sont exploitées sur l'ensemble du bassin versant ; on relève en effet la présence de 12 centrales hydroélectriques et de nombreuses piscicultures dans la partie amont.

L'Ariège a une longueur de $170 \mathrm{~km}$ et se jette dans la Garonne en amont de Toulouse. Son bassin versant couvre $3900 \mathrm{~km}^{2}$. Il est soumis à plusieurs influences climatiques : océaniques et continentales. Comme les deux précédentes rivières, les eaux de l'Ariège sont exploitées pour la production d'électricité, l'irrigation et le soutien de débits d'étiage de la Garonne.

\subsection{Stations étudiées}

Sur l'Ariège, quatre stations ont été prospectées. Sur le Gave de Pau et sur la Nive, trois stations ont été étudiées. Toutes les stations d'études sont classées dans la Zone à barbeau (Huet 1949). Les caratéristiques physiques et chimiques des 11 stations sont récapitulées dans le tableau 1.

\section{Matériel et méthodes}

\subsection{Récoltes des données}

Deux campagnes de prélèvements ont été effectuées sur chaque station de chaque rivière. L'Ariège a été échantillonnée en février et septembre 1991, lé Gave de Pau et la Nive en février 1992 et en septembre 1992. Les poissons et onze variables du milieu correspondants à leurs positions ont été relevés en de nombreux points selon la méthode de l'Échantillonnage:Ponctuel d'Abondance adaptée pour les études des adultes de poissons d'eau douce par Persat (Nelva et al. 1979, Persat \& Copp 1989) et pour les alevins par Copp (Copp \& Peñáz 1988, Copp 1989). L'échantillonnage est pratiqué par pêche électrique avec une anode alimentée par un générateur de courant continu sous 1 à 2 A et 300 à $400 \mathrm{~V}$. La progression se fait à pied selon une trajectoire perpendiculaire à la berge (semi-transect vers le large), un point en bord de rive et les autres espacés d'environ $5 \mathrm{~m}$. Dès que la prospection sur cette trajectoire est impossible (profondeur et vitesse de courant trop importante), l'opérateur reprend l'échantillonnage $5 \mathrm{~m}$ en amont selon un autre semi-transect. Un point consiste à une immersion de l'anode pendant quelques secondes. Les poissons sont immédiatement capturés à l'épuisette, déterminés, mesurés puis relâchés sur place. Le microhabitat a été évalué en utilisant les variables suivantes : la distance du point à la berge, le type de faciès d'écoulement, la présence d'abris 
Tableau 1. Caractéristiques générales des stations d'études.

Table 1. General characters of the study sites.

\begin{tabular}{|c|c|c|c|c|c|c|c|c|c|c|}
\hline & & Code & $\begin{array}{l}\text { Altitude } \\
(\mathrm{m})\end{array}$ & $\begin{array}{l}\text { Distance } \\
\text { confl. (km) }\end{array}$ & $\begin{array}{l}\text { Largeur } \\
\text { moy. }(\mathrm{m})\end{array}$ & $\begin{array}{c}\text { Temp. } \\
\text { eau }\left({ }^{\circ} \mathrm{C}\right)\end{array}$ & $\begin{array}{c}\text { Conductivité } \\
\mu \mathrm{S} / \mathrm{cm}\end{array}$ & $\begin{array}{c}\text { Temp. } \\
\text { eau }\left({ }^{\circ} \mathrm{C}\right)\end{array}$ & $\begin{array}{c}\text { Conductivité } \\
\mu S / \mathrm{cm}\end{array}$ & $\begin{array}{l}\text { Qualité } \\
\text { Eau * }\end{array}$ \\
\hline Rivières & Stations & & & & & & Eévrier & Sep & & \\
\hline \multirow[t]{4}{*}{ Ariège } & Pont du Diable & $\overline{\mathrm{A} 1}$ & 450 & 98 & 13 & 5.8 & $\overline{90}$ & 13.2 & 120 & $\overline{2}$ \\
\hline & Varilhes & A2 & 320 & 77 & 60 & 6.4 & 95 & 14.1 & 130 & 2 \\
\hline & Pamiers & A3 & 270 & 63 & 52 & 5.8 & 90 & 14.0 & 160 & 2 \\
\hline & Saverdun & A4 & 220 & 44 & 43 & 5.1 & 125 & 13.8 & 205 & 2 \\
\hline \multirow{3}{*}{ Nive } & Itxassou 1 & N1 & 35 & 34 & 40 & 9.0 & 210 & 19.0 & 250 & $1 B$ \\
\hline & Itxassou 2 & N2 & 30 & 32 & 30 & 6.8 & 235 & 17.3 & 240 & $1 \mathrm{~B}$ \\
\hline & Halsou & N3 & 15 & 22 & 55 & 11.3 & 250 & 19.0 & 240 & 2 \\
\hline Gave & Castetarbe 1 & G1 & 60 & 41 & 60 & 7.2 & 370 & 18.0 & 260 & 2 \\
\hline \multirow[t]{2}{*}{ de Pau } & Castetarbe 2 & G2 & 60 & 38 & 60 & 7.2 & 400 & 18.0 & 270 & 2 \\
\hline & Puyoo & G3 & 35 & 25 & 75 & 8.3 & 390 & 20.0 & 300 & 2 \\
\hline
\end{tabular}

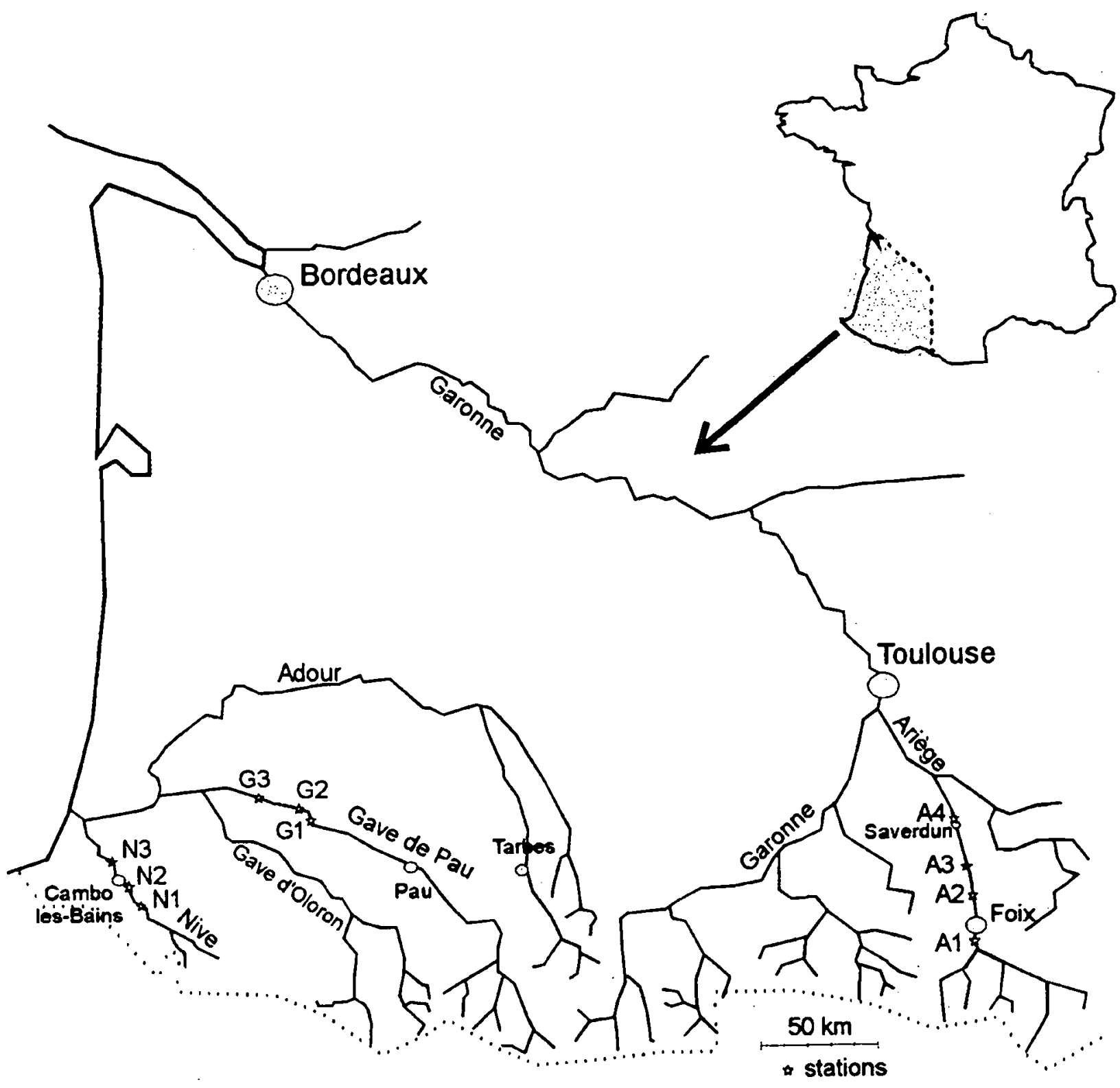

Fig. 1. Situation géographique des rivières et stations d'études. Les codes des stations sont donnés dans le tableau 1.

Fig. 1. Map of the rivers and sites under study (codes sites are given in Table 1) 
d'origine végétale, le recouvrement de blocs: $(\varnothing>20$ $\mathrm{cm})$, le recouvrement de galets $(2<\varnothing<20 \mathrm{~cm})$, le recouvrement de gravier $(2 \mathrm{~mm}<\varnothing<2 \mathrm{~cm})$, le recouvrement de sable $(50 \mu \mathrm{m}<\varnothing<2 \mathrm{~mm})$, la présence de vase $(\varnothing<50 \mu \mathrm{m})$, la présence: d'affleurement de märne ou molasse, la profondeur" de'l'eau, la vitesse du courant (à 2/3). Les différents types de granulométrie ont été déterminés en fonction de l'échelle de Cailleux (1954). La plupart des variables ont été estimées visuellement, à l'exception de la profondeur de l'eau et de la vitesse du courant qui ont été mesurées. La présence de chaque type granulométrique a été estimée en considérant le pourcentage de recouvrement par rapport à la zone échantillonnée. Cinq types de faciès d'écoulement (Malavoi 1989) ont été pris en compte : radier, rapide, plat, trou et bordure. Les abris végétaux sont regroupés selon trois modalités : absence, présence d'herbiers, présence de branches et/ou racines.

\subsection{Traitement des données}

Pour établir les relations interspécifiques et espècesvariables, les variables quantitatives ont été découpées en modalités qualitatives ou semi-quantitatives : la distance à la rive en trois modalités ( $>10 \mathrm{~m}, 5$ à $10 \mathrm{~m},<5$ $\mathrm{m})$; le recouvrement de blocs et le recouvrement de galets chacun en quatre modalités (absence, 1 à $30 \%, 31$ à $60 \%,>60 \%$ ); le recouvrement de gravier (absence, 1 à $30 \%,>30 \%$ ); la présence de vase et la présence d'affleurements de marne ou molasse en deux modalités (absence, présence); la profondeur de l'eau en cinq modalités ( $<20 \mathrm{~cm}, 21$ à $40 \mathrm{~cm}, 41$ à $60 \mathrm{~cm}, 61$ à 80 $\mathrm{cm},>80 \mathrm{~cm}$ ), la vitesse du courant en cinq modalités $\left(0 \mathrm{~cm} \cdot \mathrm{s}^{-1}, 1\right.$ à $10 \mathrm{~cm} \cdot \mathrm{s}^{-1}, 11$ à $20 \mathrm{~cm} \cdot \mathrm{s}^{-1}, 21$ à 40 $\mathrm{cm} . \mathrm{s}^{-1},>40 \mathrm{~cm} \cdot \mathrm{s}^{-1}$ ).

Les données sont réunies dans deux matrices : une matrice points par variables et une matrice points par
- espèces. Pour chaque rivière et pour chaque espèce, la distribution des individus dans les modalités des différentes variables entre hiver et été a été comparée par le test statistique de Mann-Whitney (Sprent 1992). Pour étudier les associations espèce-modalités, les profils de microhabitat de chaque espèce ont été calculés et mis sous forme de graphiques. Pour déterminer la fréquence d'occurrence des associations modalités-espèce $\left(\mathrm{Khi}^{2}\right)$, la matrice des points par variables a été croisée avec celle des points par espèces (convertie préalablement en présence (1) - absence (0)).

Le calcul des profils de microhabitat a été réalisé à l'aide de la programmathèque ADE (Chessel \& Dolédec 1993) et les graphiques de ces profils ont été effectués avec le logiciel GraphMu (Thioulouse 1990). Les profils d'habitat sont calculés comme étant la différence entre la fréquence d'une espèce dans un groupe d'échantillons ayant la même modalité pour une variable et la fréquence de cette espèce dans tous les autres points d'échantillonnage. La matrice points par espèces est croisée avec elle-même pour calculer les associations $\left(\mathrm{Khi}^{2}\right)$ espèces- espèces.

\section{Résultats}

212 à 464 points d'échantillonnage ponctuel d'abondance ont été effectués à chaque campagne et sur chaque rivière. Pour un total de 1974 points échantillonnés, 6100 poissons ont été capturés dont 3093 vairons, 1839 goujons et 1168 loches franches (Tableau 2). Les associations variables-espèces (Tableau 3) et espèces-espèces (Tableau 4) ont été étudiées pour les trois espèces sur l'Ariège et la Nive et comparées en fonction de la saison. Sur le Gave de Pau, la loche franche étant très peu représentée, seuls le vairon et le goujon ont été pris en compte (Tableau 4).

Tableau 2. Longueur totale moyenne (LT moy), maximale (LT maxi) et minimale (LT mini) d'un sous-échantillon de goujon ( $\mathrm{Gg}$ : Gobio gobio), vairon ( $\mathrm{Pp}$ : Phoxinus phoxinus) et loche franche (Bb : Barbatula barbatula) dans les trois rivières.

Table 2. Mean total length (LT moy), maximum (LT maxi) and minimum (LT mini) of a sub-sample of gudgeon (Gg : Gobio gobio), minnow ( $\mathrm{Pp}:$ Phoxinus phoxinus) and stone loach (Bb: Barbatula barbatula) from the study sites.

\begin{tabular}{|c|c|c|c|c|c|c|c|c|c|}
\hline \multirow[b]{2}{*}{ Espèces } & \multicolumn{3}{|c|}{ Ariège } & \multicolumn{3}{|c|}{ Nive } & \multicolumn{3}{|c|}{ Gave de Pau } \\
\hline & $\mathrm{Gg}$ & $\mathrm{Bb}$ & $\mathrm{Pp}$ & $\mathrm{Gg}$ & $\mathrm{Bb}$ & $P p$ & $\mathrm{Gg}$ & $\mathrm{Bb}$ & $\mathrm{Pp}$ \\
\hline LT moy $(\mathrm{mm})$ & 85,3 & 69,1 & 50,7 & 125 & 68,3 & 63,5 & 81,0 & 65 & 33,4 \\
\hline LT mini $(\mathrm{mm})$ & 30 & 30 & 20 & 50 & 30 & 25 & 25 & 60 & 30 \\
\hline LT $\operatorname{maxi}(\mathrm{mm})$ & 177 & 110 & 100 & 170 & 105 & 85 & 190 & 70 & 65 \\
\hline $\begin{array}{l}\text { Erreur standard } \\
\quad \text { (LT moy) }\end{array}$ & 1.23 & 0.89 & 0.7 & 1.58 & 7.85 & 2.52 & 2.14 & 5 & 0.91 \\
\hline $\mathrm{Nbr}$ individus & 607 & 368 & 942 & 247 & 10 & 33 & 187 & 2 & 84 \\
\hline
\end{tabular}


Tableau 3. Fréquence totale (Fqr) de chaque modalité pour chaque variable de l'environnement et fréquence de chaque espèce (Fs) dans les différents échantillons de l'Ariège, la Nive et le Gave de Pau. Les abréviations des espèces sont données dans le tableau 2.

Table 3. Total frequency (Fqr) of each category (for each environmental variable) and frequency of each species (Fs) in samples from the Rivers Ariège, Nive and Gave de Pau. Fish abbreviations are given in Table 2.

\begin{tabular}{|c|c|c|c|c|c|c|c|c|c|c|c|c|c|c|c|c|c|c|c|c|c|c|c|c|}
\hline \multirow{3}{*}{ Variables } & \multicolumn{8}{|c|}{ Ariège } & \multicolumn{8}{|c|}{ Nive } & \multicolumn{8}{|c|}{ Gave de F } \\
\hline & \multicolumn{2}{|c|}{ Far } & \multicolumn{2}{|c|}{$\overline{F s(G g)}$} & \multicolumn{2}{|c|}{$\overline{F s(B b)}$} & \multicolumn{2}{|c|}{$\overline{F s(P p)}$} & \multicolumn{2}{|c|}{$\overline{F q r}$} & \multicolumn{2}{|c|}{$F s(G g)$} & $\overline{F s(1}$ & 8b) & $\overline{F s(}$ & Pp) & $F$ & $q r$ & Fs & Gg) & $\overline{F s(}$ & & s) & Pp) \\
\hline & $\bar{h}$ & e & $\mathrm{h}$ & e & $\mathrm{h}$ & e & $h$ & e & 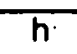 & $\sigma$ & $\bar{h}$ & $e$ & $\bar{h}$ & e & $\bar{h}$ & e & $\bar{h}$ & $e$ & $\mathrm{~h}$ & $e$ & $\mathrm{~h}$ & & $\bar{h}$ & \\
\hline & 464 & 418 & 129 & 147 & 200 & 68 & 159 & 93 & 256 & 355 & 88 & 78 & 39 & 39 & 40 & 1 & 212 & 269 & 46 & & & & 24 & 46 \\
\hline stance & & & & & & & & & & & & & & & & & & & & & & & & \\
\hline $10 \mathrm{~m}$ & 33 & 80 & 12 & 18 & 17 & 13 & 4 & 3 & 64 & 142 & 8 & 16 & 7 & 6 & 1 & 12 & 6 & 31 & 0 & & 0 & & 0 & 2 \\
\hline $0 \mathrm{~m}$ & 144 & 163 & 37 & 51 & 64 & 27 & 22 & 21 & 86 & 0 & 25 & 41 & 12 & 16 & 10 & 30 & 68 & 126 & 12 & & & & 6 & 19 \\
\hline$<5 \mathrm{~m}$ & 287 & 175 & 80 & 78 & 19 & 28 & 133 & 69 & 106 & 5 & 55 & 21 & 20 & 17 & 29 & 0 & 38 & 112 & 34 & & & & 18 & \\
\hline -iè & & & & & & & & & & & & & & & & & & & & & & & & \\
\hline radier & 212 & 193 & 54 & 75 & 01 & 40 & 91 & 54 & 115 & 127 & 34 & 18 & 20 & 18 & 10 & 13 & 73 & 8 & 14 & 4 & 1 & & 5 & 18 \\
\hline rapide & 140 & 94 & 44 & 28 & 65 & 19 & 16 & 6 & 46 & 8 & 5 & a & & & 1 & 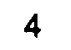 & 4 & & 1 & & & & 1 & 2 \\
\hline plat & 6 & 68 & 2 & 22 & 3 & 6 & 2 & 8 & 68 & 12 & 28 & 53 & 5 & 9 & 13 & 33 & 3 & 100 & 15 & & & & 6 & 13 \\
\hline trou & 68 & 25 & 12 & 4 & 15 & 0 & 19 & 4 & 2 & 11 & 0 & 0 & 2 & 0 & & 0 & 4 & & 1 & & & & 2 & \\
\hline rive & 38 & & 1 & 18 & 16 & 3 & 1 & 21 & 25 & 1 & 21 & & & & 4 & 1 & 58 & & 15 & & & & 0 & 13 \\
\hline ris & & & & & & & & & & & & & & & & & & & & & & & & \\
\hline ence & 355 & 355 & 90 & 115 & 53 & 57 & 75 & 65 & 195 & 258 & 53 & 29 & 29 & 25 & 15 & 14 & 189 & 245 & 40 & 7 & & & 16 & 39 \\
\hline jers & 24 & 16 & 8 & 7 & 17 & 7 & 7 & 7 & 27 & 75 & 0 & 40 & & 1 & & 0 & 10 & & 2 & & & & 4 & 1 \\
\hline leux & 85 & 47 & 31 & 25 & 30 & 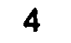 & 7 & 21 & 34 & 2 & 25 & 0 & & & 16 & 7 & 13 & & & & & & & \\
\hline & & & & & & & & & & & & & & & & & & & & & & & & \\
\hline ence & 195 & 252 & 56 & 82 & 75 & 42 & 74 & 50 & 71 & 137 & 22 & 45 & 15 & 14 & 13 & 35 & 114 & 189 & 24 & 4 & & & 15 & 40 \\
\hline$\%$ & 177 & 28 & 60 & 14 & 98 & 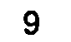 & 52 & 6 & 3 & 16 & 1 & 0 & U & 0 & 0 & 0 & 12 & 1 & 0 & & & & 0 & 0 \\
\hline $31-60 \%$ & 54 & 114 & 9 & 46 & 21 & 14 & 21 & 28 & 154 & 16 & 2 & 28 & 23 & 20 & 22 & 11 & 9 & 59 & 12 & & & & & 3 \\
\hline$>60 \%$ & 38 & 24 & 4 & 5 & 6 & 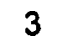 & 12 & 9 & 28 & 39 & 13 & $E$ & 1 & 5 & 5 & -5 & 37 & 20 & 10 & & & & & 3 \\
\hline & & & & & & & & & & & & & & & & & & & & & & & & \\
\hline ence & 73 & 64 & 21 & 17 & 9 & 2 & 21 & 16 & 65 & 6 & 33 & 13 & 3 & 13 & 13 & 10 & 95 & 129 & 23 & 36 & & & 13 & 17 \\
\hline $0 \%$ & 73 & 2 & 22 & 14 & 34 & 5 & 37 & 8 & 4 & 1 & 1 & 0 & 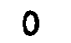 & & 0 & 0 & & & 1 & & & & | & U \\
\hline $31-60 \%$ & 115 & 309 & 22 & 110 & 53 & 50 & 49 & 66 & 157 & 18 & 48 & 30 & 24 & 17 & 25 & 3 & 6 & 31 & 15 & & & & 8 & 14 \\
\hline$>60 \%$ & 203 & 2 & 64 & 6 & 104 & 11 & 52 & 3 & 30 & 96 & 6 & 35 & 12 & 9 & 2 & 28 & 43 & 81 & 7 & 17 & & & & 15 \\
\hline ivie & & & & & & & & & & & & & & & & & & & & & & & & \\
\hline ence & 387 & 166 & 120 & 49 & 153 & 20 & 120 & 44 & 215 & 24 & 78 & 72 & 37 & 31 & 34 & 44 & 196 & 252 & 41. & 64 & & & 19 & 39 \\
\hline $0 \%$ & 17 & 9 & 4 & 46 & 11 & 20 & 12 & 21 & 4 & & ? & 0 & 0 & 2 & 0 & & 3 & & 2 & & & & 1 & 0 \\
\hline$>30 \%$ & 60 & 157 & 5 & 52 & 36 & 28 & 27 & 28 & 37 & 2 & 8 & & & & 6 & 6 & 13 & 1 & 3 & & & & & 7 \\
\hline & & & & & & & & & & & & & & & & & & & & & & & & \\
\hline absence & 396 & 354 & 94 & 123 & 165 & 60 & 115 & 66 & 218 & 30 & 68 & 68 & 37 & 35 & 33 & 46 & 179 & 171 & 38 & 4 & & & 18 & 31 \\
\hline $0 \%$ & 33 & 4 & 18 & 17 & 16 & 7 & 22 & 20 & 8 & 1 & 2 & 2 & 1 & 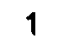 & 1 & 1 & & 11 & 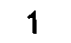 & & & & 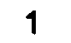 & 0 \\
\hline$>30 \%$ & 35 & 1 & 17 & 1 & 19 & 1 & 22 & 7 & 30 & 3 & 17 & 8 & 1 & 3 & 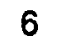 & 4 & 26 & 8 & 7 & 28 & & & & 15 \\
\hline & & & & & & & & & & & & & & & & & & & & & & & & \\
\hline ence & 436 & 402 & 119 & 140 & 190 & 67 & 139 & 86 & 248 & 347 & 82 & 76 & 37 & 37 & 35 & 48 & 179 & 24 & 35 & & & & 17 & 45 \\
\hline ce & 28 & 1 & 10 & 7 & 10 & 1 & 20 & 7 & 8 & 8 & 6 & 2 & 2 & 2 & 5 & 3 & 33 & 24 & 11 & 6 & 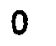 & & 7 & 1 \\
\hline & & & & & & & & & & & & & & & & & & & & & & & & \\
\hline nce & 383 & 355 & 88 & 128 & 82 & 66 & 139 & 86 & 253 & 35 & 86 & 78 & 39 & 37 & 40 & 51 & 109 & 18 & 23 & & & & 3 & 31 \\
\hline ce & 81 & 6 & 41 & 19 & 18 & 2 & 20 & 7 & 3 & 3 & 2 & 0 & 0 & 2 & 0 & 0 & 103 & 88 & 23 & 20 & 0 & 0 & 11 & 15 \\
\hline fon & & & & & & & & & & & & & & & & & & & & & & & & \\
\hline $\mathrm{cm}$ & 32 & 3 & 7 & 9 & 12 & 10 & 15 & 9 & 2 & & 9 & 11 & $\varepsilon$ & & 3 & & & & 3 & & & & 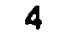 & 17 \\
\hline $\mathrm{cm}$ & 157 & 1 & 49 & 7 & 76 & 42 & 67 & 55 & 101 & 1 & 4 & 31 & 17 & 19 & 8 & 22 & 80 & 91 & 20 & & & & 11 & 15 \\
\hline $\mathrm{cm}$ & 146 & 11 & 4 & 45 & 72 & 12 & 43 & 16 & 79 & 89 & 29 & 25 & 9 & 6 & 14 & 16 & 9 & & 15 & 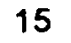 & & & 3 & 9 \\
\hline $61-80 \mathrm{~cm}$ & 95 & 4 & 23 & 13 & 35 & 3 & 23 & 7 & 45 & 58 & 12 & 10 & 5 & 2 & 3 & 7 & 9 & 3 & 7 & 0 & & & 5 & 1 \\
\hline$>80 \mathrm{~cm}$ & 34 & 33 & 5 & 7 & 5 & 1 & 11 & 6 & 10 & 10 & 4 & 1 & 0 & 1 & 2 & $v$ & 16 & 50 & 1 & 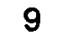 & 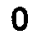 & 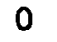 & 6 & 0 \\
\hline & & & & & & & & & & & & & & & & & & & & & & & & \\
\hline $5-1$ & 11 & 6 & 2 & 2. & 38 & 5 & & 2 & 2 & & & $\varepsilon$ & & 10 & 1 & & & & 10 & & & & & 9 \\
\hline & 68 & 4 & 25 & 16 & 28 & 9 & 30 & 2 & 2 & 2 & 14 & 3 & 4 & 1 & 6 & 2 & 48 & 3 & 14 & 13 & 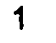 & & 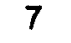 & 12 \\
\hline-1 & 77 & 7 & 21 & 34 & 32 & 16 & 41 & 25 & 36 & 5 & 3 & 15 & 10 & $\epsilon$ & 10 & 8 & 48 & 36 & 12 & 0 & 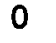 & & 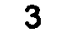 & 8 \\
\hline$s-1$ & 118 & 160 & 37 & 50 & 59 & 21 & 25 & 19 & 90 & 13 & 26 & 45 & 14 & 13 & 13 & 32 & 58 & 103 & 8 & 27 & 1 & 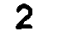 & 4 & 14 \\
\hline $\mathrm{cm} \mathrm{c}_{-1}$ & 87 & 75 & 18 & 25 & 43 & 17 & 0 & 2 & 83 & 121 & 15 & 7 & 10 & 6 & 0 & 2 & 14 & 62 & 2 & 10 & 0 & 0 & 1 & 3 \\
\hline
\end{tabular}


Tableau 4. Associations $\left(\mathrm{Khi}^{2}\right)$ entre les espèces sur chaque rivière et chaque date de prélèvement. Les associations significatives sont indiquées avec un astérisque $\left(^{* * *}=\mathrm{P}<\right.$ $\left.0.005,{ }^{*} * \mathrm{P}<0.01,{ }^{*}=\mathrm{P}<0.05\right)$.

Table $4 . \mathrm{Khi}^{2}$ associations between species of the three rivres under study. Significant $\left(\mathrm{Khi}^{2}\right.$ associations are indicated with an asterisk $\left(^{* * *}=\mathrm{P}<0.005, * *=\mathrm{P}<0.01,{ }^{*}=\mathrm{P}\right.$ $<0.05$ ).

\begin{tabular}{|c|c|c|c|c|c|c|c|c|c|}
\hline \multirow[t]{2}{*}{$\overline{\text { Saison }}$} & & \multicolumn{3}{|c|}{ Ariège } & \multicolumn{3}{|c|}{ Nive } & \multicolumn{2}{|c|}{ Gave de Pau } \\
\hline & & $\overline{\mathrm{Gg}}$ & $\overline{\mathrm{Bb}}$ & $\mathrm{Pp}$ & $\overline{G g}$ & $\overline{\mathrm{Bb}}$ & $\overline{\mathrm{Pp}}$ & $\mathrm{Gg}$ & $\mathrm{Pp}$ \\
\hline & $\mathrm{Gg}$ & - & $\star$ & * & - & - & $\star \star \star$ & - & $\star \star \star \star$ \\
\hline Hiver & $\mathrm{Bb}$ & & - & $\star \star \star \star$ & & - & - & - & - \\
\hline & $P p$ & & & - & & & - & & - \\
\hline & $\mathrm{Gg}$ & - & - & $\star \star \star \star$ & - & & $\star \star \star$ & - & 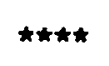 \\
\hline Eté & $\mathrm{Bb}$ & & - & * & & - & $\star \star \star \star$ & - & - \\
\hline & $\mathrm{Pp}$ & & & - & & & - & & - \\
\hline
\end{tabular}

\subsection{Associations espèces - variables de l'habitat et variations saisonnières}

\subsubsection{Le vairon (Fig. 2)}

Sur l'Ariège, les habitats ponctuels colonisés par le vairon en hiver ont une faible profondeur; une vitesse nulle à faible; un substrat mixte : galet, gravier, sable et vase et sont plutôt en bord de rive. Le vairon évite le chenal central de la rivière et la présence de marne. En été, les même caractéristiques d'habitat sont observés sauf les asșociations avec les galets et le gravier.

On observe des différences significatives entre l'hiver et l'été pour quatre des onze variables du microhabitat du vairon: une distance à la berge plus faible, les abris végétaux moins recherchés, le recouvrement de galets et de gravier indifférent en été.

Sur la Nive, en. hiver, le vairon ne montre d'associations significativeș que pour une vảriable : la présence d'affleurements de marne. Des différences significatives entre saisons sont observées pour cinq yariables: la distance à la berge, le type de faciès, le recouvrement de bloc et de galets et la vitesse du courant. En effet, en été, les microhabitats colonisés par le vairon sont caractérisés par un fort recouvrement de galets, l'absence de blocs, une vitesse de courant nulle ou moyenne et la présence d'abris (herbiers, branches/racines). Le vairon préfère les faciès de type plat.

Sur le Gave de Pau, en hiver, les habitats ponctuels colonisés par le vairon possèdent un fort recouvrement de gravier avec des abris (herbiers, branches/racines). Les changements significatifs entre les saisons d'étiage se situent au niveau de 6 variables du microhabitat. En été, le vairon préfère les herbiers pour abris, les faibles profondeurs et les vitesses nulles à faibles. Il évite les forts recouvrements de blocs et la présence de vase.

\subsubsection{Le goujon (Fig. 3)}

Sur l'Ariège, en hiver, le goujon est associé avec les variables granulométriques. Il préfère les microhabitats à forts recouvrements de sable et de marne. Par contre, il évite les blocs et le gravier. En été, il recherche les abris sur un substrat de type galet et gravier. Ces microhabitats sont situés dans la frange rivulaire de la rivière. Les associations avec la profondeur, la vitesse du courant, le recouvrement de galets, gravier et sable, le type d'abri sont significativement différentes entre les deux étiages. En effet, en été, le goujon préfere les abris de type branches/racines, sur un substrat mixte de galets, gravier et sable. Il évite les affleurements de marne et les fortes profondeurs.

Sur la Nive, en hiver, le goujon n'est associé significativement avec aucune variable du microhabitat. En été, il colonise les habitats avec des abris (herbiers, branches/racines), un substrat de galet et des vitesses de courant nulles ou moyennes. Il évite la présence de blocs. Les variations significatives entre saisons sont la distance à la rive, le recouvrement de blocs et de galets et la vitesse de courant.

Sur le Gave de Pau comme sur la Nive, le goujon n'est associé avec aucune variable en hiver. En été, il recherche les abris (surtout les herbiers) avec un fort recouvrement de blocs. La distance à la rive, le recouvrement de sable, de vase et de marne et la vitesse de courant sont les quatre variables où des variations significatives sont observés entre saisons. 

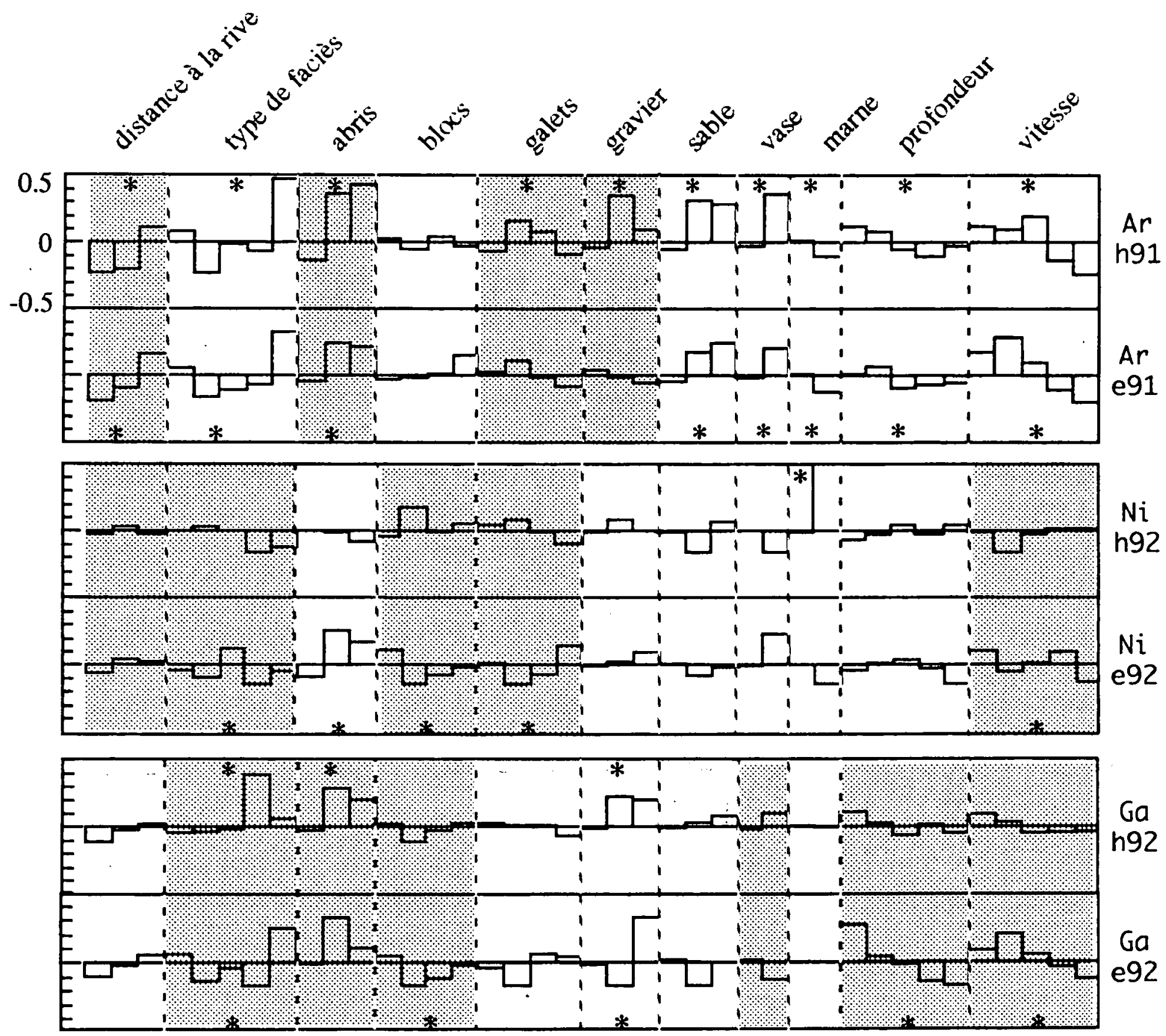

Fig. 2. Profils de microhabitat et association $\left(\mathrm{Khi}^{2}\right)$ entre le Vairon (Pp) et les variables dans l'Ariège (Ar), la Nive (Ni) et le Gave de Pau (Ga) à chaque date d'échantillonnage (h91 = hiver 1991, e91 = été 1991, h92 = hiver 1992, e92= été 1992). Chaque histogramme représente la différence entre la fréquence des espèces dans un groupe d'échantillons ayant la même modalité pour une variable de l'environnement et la fréquence des espèces dans tous les échantillons. Les relations significatives entre espèces et variables sont indiquées avec un astérisque $(*=\mathrm{P}<0.05)$ et entre espèces et modalités par une valeur approchant $10.5 \mathrm{l}$. Les rectangles de couleur grise désignent les variations significatives des associations espèces variables entre hiver et été. Voir le tableau 3 pour l'identification des différentes modalités par variables.

Fig. 2. Microhabitat profiles and chi-square associations for minnow (Pp) for Ariège river (Ar), Nive river (Ni) and Gave de Pau river $(\mathrm{Ga})$ and each sampling date $(\mathrm{h} 91=$ winter 1991 , e91 = summer 1991, h92 = winter 1992, e92 = summer 1992). Each histogram represents the difference between the frequency of that species in the groupe of samples having that categories of environmental variable and the frequency of that species in all samples. Significant deviations from expected between species and variables are indicated with an asterisk $\left(^{*}=\mathrm{P}<0.05\right)$, between species and individual categories by values approching 10.51 and between winter and summer by the greyish rectangle. 

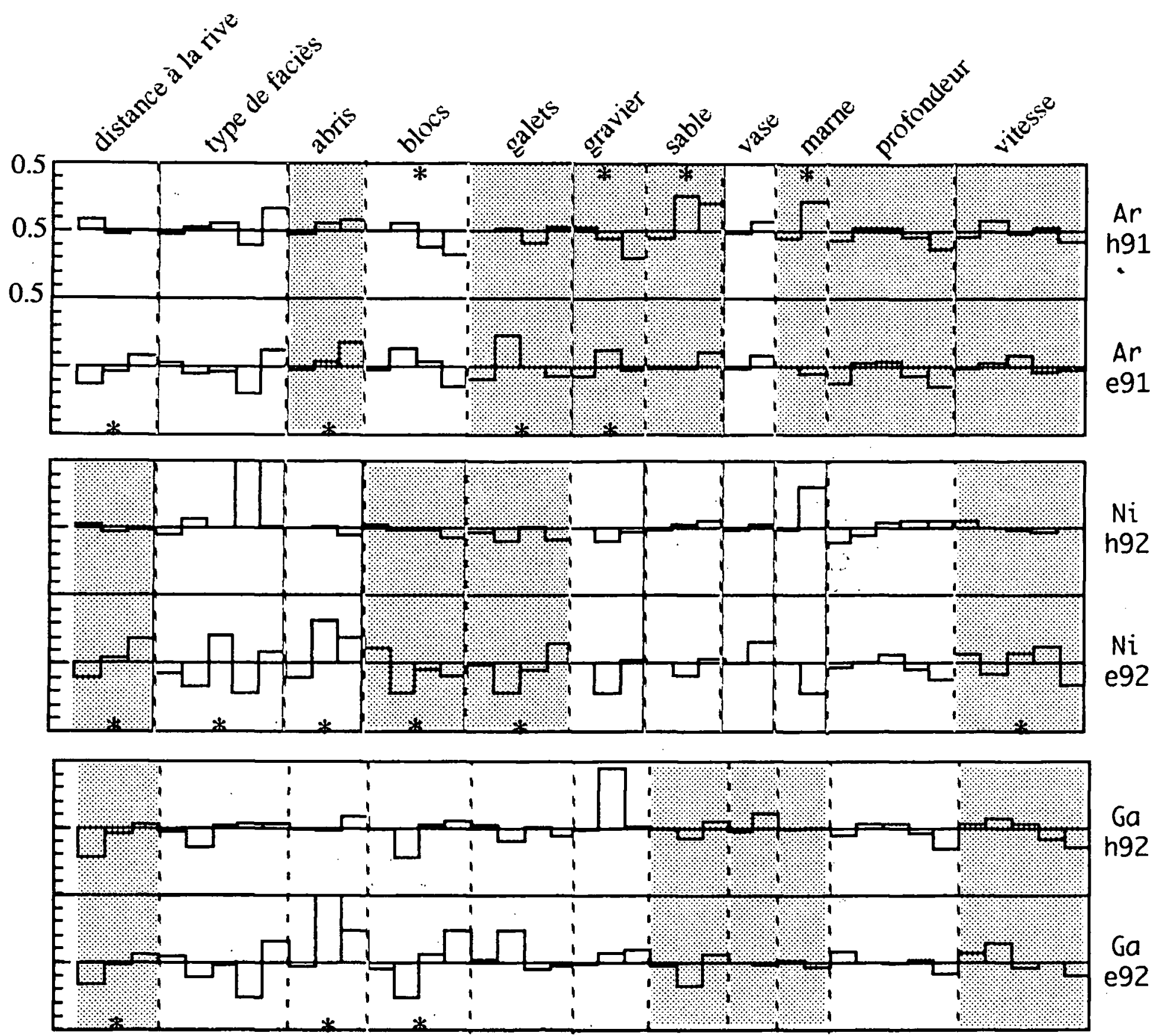

Fig. 3. Profils de microhabitat et association $\left(\mathrm{Khi}^{2}\right)$ entre le Goujon $(\mathrm{Gg})$ et les variables dans l'Ariège (Ar), la Nive (Ni) et le Gave de Pau (Ga) à chaque date d'échantillonnage (h91 =hiver 1991, e91 = été 1991, h92 = hiver 1992, e92 = été 1992).

Fig. 3. Microhabitat profiles and chi-square associations for gudgeon $(\mathrm{Gg})$ for Ariège river (Ar), Nive river ( $\mathrm{Ni})$ and Gave de Pau river $(\mathrm{Ga})$ and each sampling date $(\mathrm{h} 91$ = winter 1991, e9l = summer 1991, h92 $=$ winter 1992, e92 = summer 1992).

\subsubsection{La loche franche (Fig.4)}

Cette espèce étant très peu représentée sur. le Gave de Pau, les calculs ont été effectués à partir des données récoltées sur l'Ariège et la Nive.

Sur l'Ariège, les habitats colonisés en hiver par la loche ont une profondeur moyenne de 20 à $60 \mathrm{~cm}$, des herbiers et un substrat mixte de blocs, de galets et de gravier. Ces habitats sont situés dans des faciès courant (radier, rapide et plat). La loche évite les affleurements de marne et les profondeurs importantes (trou). En été, la loche montre des associations significatives avec les mêmes variables, excepté le recouvrement de blocs et de gravier. Les associations avec le recouvrement de galets et de gravier, la profondeur et la distance à la berge diffèrent significativement entre les périodes d'étiage.

Sur la Nive, la loche est en hiver associée significativement avec la vitesse de courant. En effet, elle évite 

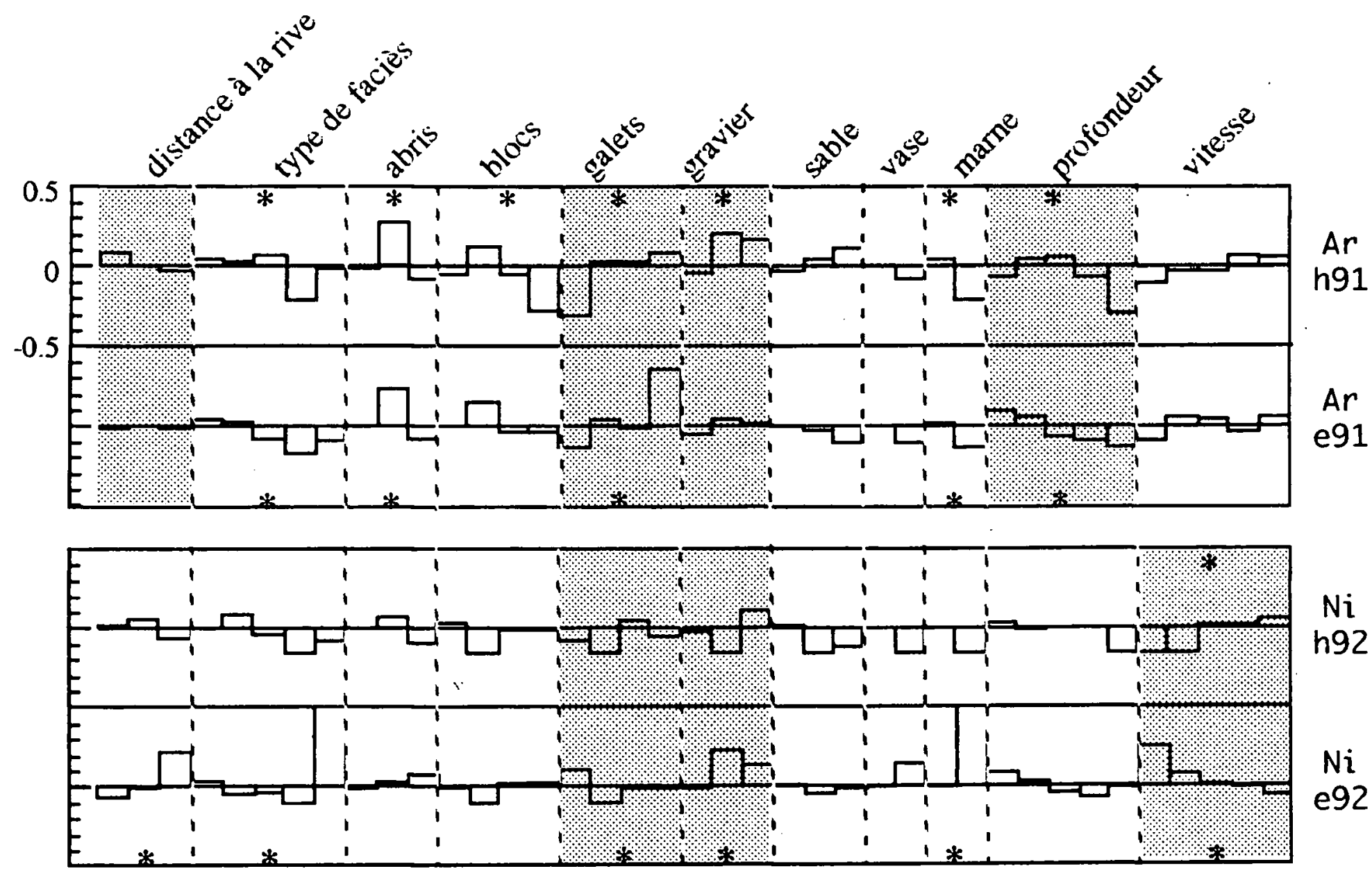

Fig. 4. Profils de microhabitat et association $\left(\left(\mathrm{Khi}^{2}\right)\right.$ entre la loche franche $(\mathrm{Bb})$ et les variables dans l'Ariège (Ar) et la Nive (Ni) à chaque date d'échantillonnage (h91 =hiver 1991, e91 = été 1991, h92 = hiver 1992, e92 = été.1992).

Fig. 4. Microhabitat profiles and chi-square associations for stone loach $(\mathrm{Bb})$ for Ariège river $(\mathrm{Ar})$ and Nive river $(\mathrm{Ni})$ and each sampling date $(\mathrm{h} 91$ = winter 1991, e91 = summer 1991, h92 = winter 1992, e92 = summer 1992).

les vitesses nulles ou faibles. En été, cette espèce affectionne les habitats avec un recoürement de gravier et de marne. Elle évite la présence de galets.,Les associations avec le recouvrement de galets, le recouvrement de gravier et la vitesse du courant diffèrent significativement entre les saisons

\subsection{Associations interspécifiques}

Sur les trois rivières et à chaque campagne, le goujon et le vairon sont toujours associés de manière significative. L'association entre la loche et le vairon est observée sur l'Ariège quelle que soit la campagne et sur la Nive en période d'étiage estival. Le goujon et la loche ne sont associés significativement qu'en hiver sur l'Ariège.

\section{Discussion}

Les variables du microhabitat sélectionnées dans cette étude sont celles qui sont retenues le plus souvent pour la description de l'habitat des poissons (Gorman \& Karr 1978, Angermeier 1987). Par rapport aux nombreuses études effectuées sur le Rhône et ses annexes, le repérage visuel des poissons échantillonnés et la composition du substrat a été en tout point possible du fait de la faible profondeur et de l'absence de turbidité. L'échantillonnage ponctuel d'abondance est donc approprié pour l'étude des relations poissons/habitat dans les trois rivières.

Sur l'Ariège, les trois espèces montrent plus de relations avec le milieu que sur les deux autres rivières. Sur le Gave de Pau et la Nive, les espèces sont significativement associées avec un grand nombre de variables du microhabitat à l'étiage estival.

On observe que les trois espèces colonisent plus souvent les habitats proches $(<10 \mathrm{~m})$ de la rive. Toutefois, la nature du substrat apparaît comme le facteur déterminant de la microrépartition des trois espèces dans les trois rivières. En effet, les substrats composés de galets 
et gravier sont colonisés par le vairon, le goujon et la loche franche dans $60 \%$ des cas. Une étude réalisée en période d'étiage estival (Septembre) sur la Basse Nivelle par Neveu (1981) montre que la loche franche est présente dans les faciès à dominance de cailloux et de galets. Cette même répartition a été souvent observée (Smyly 1955, Spillmann 1961, Copp 1992).

Une étude en laboratoire par Jacobsen (1979) sur le vairon révèle que les adultes montrent des préférences significatives pour un substrat de type cailloux $(2 \mathrm{~mm}$ $<\varnothing<50 \mathrm{~mm}$ ) par rapport à un substrat de type sable. Cette nette préférence a été également observée dans un lac norvégien (Lien 1981).

La présence d'abris végétaux semble importante dans la répartition des trois espèces. Elles ne s'écartent guère au delà de 10 mètres par rapport à la berge. C'est dans cette frange que les poissons peuvent trouver des herbiers, des branchages ou des systèmes racinaires. Smyly (1955) et Lien (1981) ont observé que la loche franche et le vairon sont souvent associés à ce type de couvert.

Les préférences observées au niveau du choix du type de substrat sont à mettre en relation avec le comportement benthique des espèces, surtout le goujon et la loche, et leur aptitude à se cacher sous les cailloux. Ces structures complexes peuvent, pour les petites espèces servir de zones refuge face aux vitesses de courant trop élevées et face à la prédation par d'autres espèces comme la truite commune (Salmo trutta) présente dans tous les secteurs étudiés. La présence d'un abri favorise la colonisation d'un habitat puisqu'il permet de réduire la déperdition d'énergie liée à la lutte contre le courant (Fausch 1984) et augmente la sécurité des individus en cas de danger. De plus, le substrat constitue un important facteur dans la productivité en invertébrés et en périlithon, et donc dans le choix des zones d'alimentation pour les trois espèces qui se nourrissent d'invertébrés. Les substrats stables et non colmatés représentent les conditions les plus favorables à la colonisation des invertébrés. La stabilité du substrat, dans le cas des espèces strictement benthiques comme la loche franche, peut être un facteur limitant des plus importants puisque ces espèces nagent peu et ont un comportement très sédentaire avec un espace vital limité aux anfractuosités du substrat (Pouilly 1994).

En période de reproduction, les trois espèces présentent des préférences dans le choix du substrat. Balon (1975) propose et définit des guildes de reproduction des espèces. Le vairon, espèce lithophile, dépose ses oeufs sur blocs, galets ou graviers. Le goujon et la loche franche, espèces psammophiles, dispersent leurs oeufs sur fonds sableux ou sur petites racines surplombant du sable.

Ces observations montrent que toute perturbation naturelle ou provoquée du substrat et des abris végétaux aura un impact sur les trois espèces. Ces espèces accompagnatrices de la truite participent en effet au bon fonctionnement de l'écosystème. En aucun cas, leur étude ne doit être négligée car elles donneront toujours une image du dysfonctionnement ou des perturbations du milieu. La rapidité de leur réponse à des bouleversements du milieu est un élément important dans le choix des espèces cibles pour des études d'impacts.

\section{Travaux cités}

Angermeier P.L. 1987. - Spatiotemporal variation in variation in habitat selection by fishes in small Illionois streams. In : Matthews W.J. Heins D.C.(ed), Community and Evolutionary Ecology of North American Stream Fishes. Norman : University of Oklaoma Press : $52-60$

Balon E.K. 1975. - Reproductive guilds of fishes : A proposal and definition. J. Fish. Res. Board. Can., 32 : 821-864.

Baltz D.M., Moyle P.B. \& Knight N.J. 1982. - Competitive interactions between benthic stream fishes, riffle sculpin, Cottus gulosus, and speckled dace, Rhinichthys osculus. Can. J. Fish. Aquat. Sci., 39: 1502-1511.

Baran P. 1995. - Analyse de la variabilité des abondances de truites communes (Salmo trutta L.) dans les pyrénées centrales françaises - Influence des échelles d'hétérogénéité de l'habitat. Thèse de $3^{\text {2n }}$ cycle. INP ENSA Toulouse : $147 \mathrm{p}$.

Bean C.W. \& Winfield I.J. 1989. - Biological and ecological effects of a Ligula intestinalis (L.) infestation of the gudgeon, Gobio gobio (L.), in the Lough Neagh, Northern Ireland. J. Fish Biol., 34 : 135-147.

Cailleux A. 1954. - Limites dimensionnelles des noms des fractions granulométriques. Bull. Soc.Géol.F; $4: 643-646$.

Chessel D. \& Dolédec S. 1993. - ADE Version 3.6 : HyperCardO Stacks and QuickBasic Microsoft@ Programme Library for the Analysis of Environnemental Data. Université de Lyon I, 43 bd du 11 nov. 1918, 69622 Villeurbanne Cedex, France

Copp G.H. 1989. - Electrofishing for fish larvae and 0+ juveniles : equipement modifications for increased efficiency with short fishes. Aquacult. \& Fish. Mgmt., $20: 177-186$.

Copp G.H. 1992. - An empirical model for predicting microhabitat of $0+$ juvenile fishes in a lowland river catchment. Oecologia (Berlin), $91: 338-345$

Copp G.H. \& Peñáz M. 1988. - Ecology of fish spawning and nursery zones in the flood plain, using a new sampling approach. $H y$ drobiologia, $169: 209-224$.

Copp G.H., Warrington S. \& Q. de Bruine 1994. - Comparison of diet in bullhead, Cottus gobio and stone loach, Barbatula barbatula in a small english lowland river. Folia Zool., 43(2): 171-176.

Cui Y. \& Wootton R.J. 1989. - Grouping fails to affect the growth and energy budget of a cyprinid, Phoxinus phoxinus (L.). J. Fish Biol. 35: 795-798.

Fausch K.D. 1984.- Profitable stream positions for salmonids : relating specific growth rate to net energy gain. Can.J. Zool., 62 : 441-451.

Gorman O.T. \& Karr J.R. 1978. - Habitat structure and streams fish communities. Ecology, $59: 507-515$ 
Grossman G.D. \& Freeman M. C. 1987. - Microhabitat use in a stream fish assemblage. J. Zool., London, $212: 151-176$.

Heggenes J. 1988 - Substrate preferences of brown trout fry (Salmo trutta) in artificial stream channels. Can. J. Fish. Aquat. Sci., $45: 1801-1806$

Huet H., 1949. - Aperçu des relations entre la pente et les populations piscicoles des eaux courantes. Rev. Suisse Hydrol., IX : 332351.

Jacobsen O.J. 1979. - Substrate preference in the minnow (Phoxinus phoxinus L.). Pol. Arch. Hydrobiol., 26(3): 371-378.

Kennedy M. 1981. - Individual variation in homing tendency in the european minnow, Phoxinus phoxinus (L.). Anim. Behav., 29 : $621-625$.

Lien L. 1981.- Biology of the minnow Phoxinus phoxinus and its interactions with brown trout Salmo trutta in $\emptyset$ vre Heimdalsvatn, Norway. Holart. Ecol. $4: 191-200$.

Lobón-Cerviá J., Montañes C. \& De Sostoa A. 1991. - Influence of environnement upon the life history of gudgeon, Gobio gobio (L.) : a recent and successful colonizer of the Iberian Peninsula. J. Fish Biol. $39: 285-300$

Malavoi J.R. 1989. - Typologie des faciès d'écoulement ou unités morphodynamiques d'un cours d'eau à haute énergie. Bull. $F r$ : Pêche Pisc. 315: 189-210.

Mann R.K.H. 1980. - The growth and reproductive strategy of the gudgeon, Gobio gobio (L.), in two hard-water rivers in southern England. J. Fish Biol., 17 : 163-176.

Mills C.A. 1988. - The effect of extreme northerly climatic conditions on the life history of the minnow, Phoxinus phoxinus ( L.). J. Fish Biol. 33 : 545-561.

Nelva A., Persat H. \& Chessel D. 1979. - Une nouvelle méthode d'étude des peuplements ichtyologiques dans les grands cours d'eau par échantillonnage ponctuel d'abondance. C. R. Acad. Sci. Paris t., 289 Série D : 1295-1298.

Neveu A. 1980 - Densité et microrépartition des espèces de poissons dans la Basse Nivelle, petit fleuve côtier des Pyrénées atlantiques. Bull. Fr. Pisci. 280: 86-103.
Persat H. \& Copp G. H. 1989. - Electrofishing and Point Abundance Sampling for the ichtyology of large rivers. In : COWX, I. (ed.). Developments in Electrofishing. Fishing News Books. Blackwell Scientific Publishing, Oxford, 203-215.

Przybylski M. \& Bañbura J. 1989. - Feeding relations between the gudgeon (Gobio gobio L.) and the stone loach (Noemacheilus barbatulus L.). Acta Hydrobiol., 31 (1/2) : 109-119.

Pouilly M. 1994. - Relations entre l'habitat physique et les poissons des zones à cyprinidés rhéophiles dans trois cours d'eau du Bassin Rhodanien : vers une simulation de la capacité d'accueil pour les peuplements. Thèse $3^{\text {imx }}$ cycle. Univ. C. Bernard. Lyon I : $256 \mathrm{p}$.

Shirvell C.S. \& Dungey R.D. 1983. - Microhabitats chosen by brown trout for feeding and spawning in rivers. Trans. Am. Fish. Soc., $112: 355-367$.

Smyly W. J. P. 1955. - On the biology of the stone-loach Noemacheilus barbatulus (L.). J. Anim. Ecol., 24 : 167-186.

Spillmann C.J. 1961. - Poissons d'eau douce. Faune de France. Editions Paul le Chevalier, Paris, 303 p.

Sprent P. 1992. - Pratique des statistiques nonparamétriques. INRA éditions, $294 \mathrm{p}$.

Stott B. \& Buckley B.R. 1979. - Avoidance experiments with homing shoals of minnows, Phoxinus phoxinus in a laboratory stream channel. J. Fish Biol., 14 : 135-146.

Thioulouse J. 1990. - MacMul and GraphMu : two Macintosh programs for the display and analysis of multivariate data. Computers and Geosciences, 16, $8: 1235-1240$.

Welton J.S., Mills C.A. \& Pygoll J.R. 1991. - The effect of interaction between the stone loach Noemacheilus barbatulus (L.) and the bullhead Cottus gobio (L.) on prey and habitat selection. $H y$ drobiologia, $220: 1-7$.

Wheeler A. 1992. - A list of the common and scientific names of fishes of the British Isles, J. Fish. Biol., 41 (suppl. A) : 1-37.

Wootton R.J., Allen J.R.M. \& Cole S.J. 1980. - Effect of body weight and temperature on the maximum daily food consumption of Gasterosteus aculeatus L. and Phoxinus phoxinus (L.) : selecting an appropriate model. J. Fish Biol., 17 : 695-705. 